\title{
In situ molecular imaging of proteins in tissues using mass spectrometry
}

\author{
William M. Hardesty and Richard M. Caprioli \\ Mass Spectrometry Research Center, Vanderbilt University, 465 21st Ave S. 9160 MRB III, \\ Nashville, TN 37232, USA
}

\section{Introduction}

Knowledge of the location and abundance of proteins in different cellular regions of tissue is critical to understanding their biological functions. Matrix-assisted laser desorption/ ionization imaging mass spectrometry (MALDI IMS) combines parallel, high-throughput molecular analysis with location-specific information for the characterization of protein distributions directly from thin sections of intact biological tissue [1]. This technology enables discovery in biological systems and has unique advantages because it: (1) generates molecular-weight-specific data, (2) requires no target-specific reagents (such as antibodies), (3) can sample intact large proteins of $100 \mathrm{kDa}$, (4) is amenable to high-throughput protocols since it is capable of acquiring over 1,000 spectra per second with hundreds of proteins monitored in parallel in a single experiment, and (5) incorporates relatively simple and robust sample preparation. Moreover, MALDI IMS is complementary with other proteomic strategies like 2D gel electrophoresis and LC-MS/MS analysis since these require tissue homogenization that destroys spatial information and obscures small differences between regions of cells in tissue.

MALDI uses a laser to desorb and ionize molecules in a sample that have been cocrystallized with a suitable matrix, typically a small aromatic molecule. Sample preparation is straightforward: a frozen section of tissue is cut 5- to 20- $\mu \mathrm{m}$ thick, thawmounted onto a MALDI target plate, matrix is applied directly to the tissue and allowed to air-dry. Matrix may be deposited using an automated matrix spotter (ca. 120-pL droplets) or by using an automated spray device or simple aerosol sprayer [2]. The method of matrix application and solvent system chosen may have an effect on the quality of MS spectra and these should be optimized for each project. Once the matrix is applied, a complete mass spectrum is acquired at specific $(x, y)$ coordinates directly from the tissue, recording both the sampling location and ions detected. This is repeated in an ordered array across the substrate, where the sampling positions become 'pixels' that are compiled to generate a picture or image for each molecule detected. Any given mass-to-charge $(\mathrm{m} / \mathrm{z})$ signal in the spectrum can be displayed with its relative intensity over the entire array, giving a density map of that compound in the array area. Hundreds of such pictures or images can be generated from a single acquisition experiment over such an array.

The power of MALDI IMS technology lies in its capability to reliably combine protein data with specific cellular regions within the tissue, e.g., in the case shown in Fig. 1 for metastatic tumor cells and normal lymphocytes (laboratory, U. d. f. t. a. s, unpublished). The affected human lymph node shown is primarily composed of normal lymphocytes, with 
small pockets of metastatic melanoma tumor cells. Manual extraction of the tumor cells is difficult, and homogenization of the tissue would dilute the unique protein pool of the tumor cells. MALDI IMS images shown in Fig. 1 reveal several proteins observed at higher intensity in tumor cells $(\mathrm{m} / \mathrm{z} 10,091,10,627$, and 11,643$)$ and proteins with high intensity specific to lymphocytes $(\mathrm{m} / \mathrm{z}, 11,307,13,375$, and 15,327$)$. The matrix array shown was robotically deposited at $150-\mu \mathrm{m}$ spacing and in this case determines the lateral imaging resolution. Although spray deposition images are routinely acquired below $50-\mu \mathrm{m}$ lateral resolution, robotic droplet deposition generally produces higher quality spectra. For reference, the average diameter of mammalian cells is ca. $10 \mu \mathrm{m}$.

Correlation of an observed $\mathrm{m} / \mathrm{z}$ value in the MALDI spectra with a specific protein is done in the following manner. Typically, a small portion of the tissue is homogenized and proteins are isolated by HPLC and their molecular analytes are verified by MALDI MS. This isolate is further purified by gel electrophoresis, the gel band containing the protein of interest is removed, in-gel digested with trypsin, followed by LC-MS/MS analysis of the resulting peptides and comparison with a protein database. When possible the recovered theoretical molecular weight should match that determined experimentally, taking into account any loss of methionine, cystine disulfide bridges, acetylations, or other modifications from the protein database.

Interest in MALDI imaging technology has grown among commercial vendors, who are now offering mass spectrometers with imaging capability, automated devices for matrix application, such as liquid jet dispensers and chemical printers, acoustic-driven spotters, and controlled spray deposition machines, and software/hardware solutions for MALDI image acquisition and data processing. Automated matrix application, either by picoliter droplet or spray coating, serves to reduce variations in matrix crystallization, deposition volumes, and drying times leading to a significant improvement in reproducibility, speed, and accuracy of matrix deposition [2]. The laser repetition rate is a crucial component for timely data acquisition, as many imaging experiments have 2,000 or more spots to be acquired. Commercial MALDI instruments are equipped with lasers having repetition rates of $200 \mathrm{~Hz}$ or more (i.e., 200 full spectra are acquired per second), with 1-kHz lasers soon to be available. For comparison, full data acquisition of an image with 2,000 spots or pixels and using 300 shots per spot would take nearly $1 \mathrm{~h}$ with a $200-\mathrm{Hz}$ laser compared with a bit over 12 min with a $1-\mathrm{kHz}$ laser.

\section{Applications}

MALDI IMS has been employed as an imaging technology in a wide variety of applications from the analysis of small molecules such as drugs and endogenous metabolites to macromolecules such as high molecular weight proteins. In a recent example, studies utilizing MALDI IMS of a mouse model of Parkinson's disease revealed a significant decrease in PEP-19 expression levels in the striatum after administration of the drug MPTP [3]. This finding was further corroborated by measuring both mRNA expression levels and LC-MS/MS analysis of the region. In two separate studies, investigators demonstrated 3D MALDI images of the brain, detailing the workflow and reproducibility of MALDI IMS in multiple serial tissue sections [4,5]. In addition, protein-specific 3D images of mouse brains were shown in complement with MRI 3D imaging technology [6]. MALDI IMS has also been employed to acquire protein and drug metabolites across an entire rat sagital section, revealing organ-specific protein signals and localization of the drug olanzapine and its metabolites [7].

Although many processes occur within clearly defined borders in tissue, MALDI IMS has also been used to track changes that proceed gradually from one area to another. In one 
investigation of bovine lenses, IMS revealed the gradient change in $\alpha \mathrm{A}$-crystallin, $\alpha \mathrm{B}$ crystallin, and the degradation products and phosphoralated forms of both $\alpha$-crystallins from the new growth cells on the lens periphery toward the aged cells in the center of the lens [8]. This process of protein degradation as the cells age has been implicated in catarogenesis. In this case, MALDI IMS measurements linked protein degradation and modification to the age of the cells by their spatial location within the lens. In another developing investigation, the technology was useful for exploration of the protein dynamics across the margin between tumor and normal tissue, a challenge to protocols that rely on tissue homogenization and extraction [9]. The extent to which tumor regions exert influence and 'project' into the surrounding tissue may aid pathologists and surgeons in setting guidelines for resection margins in various cancers and provide information on the nature of tumor growth and invasion.

The unique capabilities of IMS also enable in situ proteomics to be carried out, such as the use of chemical and enzymatic reactions prior to matrix application. For example, enzymatic digestion using trypsin was carried out in an ordered array of microspots across sections of rat brain with subsequent matrix application directly onto the digested spots [10]. The spatial selectivity of the digestion was demonstrated where multiple unique digested peptides from one brain section display the same distribution as the intact parent protein in a serial section. This work demonstrates significant improvements in the sensitivity, the number of observed analytes, the detection of larger proteins, and importantly allows unambiguous MS/MS identification directly from the tissue. Enzymatic digestion prior to IMS has also been applied to the analysis of formalin-fixed paraffin-embedded (FFPE) tissue [11]. FFPE is the predominant method for tissue preservation and storage; however, the procedure cross-links proteins, preventing classical proteomic analysis. An antigen retrieval step is employed to partially reverse the effects of formalin fixation followed by tryptic digestion and MALDI IMS of the peptides. This work opens the way to apply proteomic analysis to vast libraries of preserved tissue sections, opening the door to the discovery of more robust biomarkers of disease.

Many investigators wish to apply in situ IMS capabilities to a targeted analysis of specific cell groups for comparison. This approach, termed profiling, pools data of two or more cell types for statistical characterization of proteome differences. The data can then be mined for significant proteins and the proteomic features incorporated into models of disease class prediction, survival markers, or any number of other biomarker analyses. Applications for this technology have examined proteomic features that can classify high and low grade gliomas and differentiate patient survival in grade IV gliomas [12]. An automated workflow to target specific cells with precise matrix deposition has been detailed, showing improvements in the ability to target small cell groups, increased throughput of profiling, and improved data for subsequent statistical evaluation [13]. Figure 2 demonstrates the results from profiling 48 human stage III melanoma tumors and 18 control lymph nodes (laboratory, U. d. f. t. a. s, unpublished). The specific tumor cells (red spots) and normal lymphocyte cells (green spots) were found by examination of a serial hematoxylin and eosin $(\mathrm{H} \& \mathrm{E})$ stained section and then targeted by automated matrix deposition, Fig. 2a. Spectra across all patients are compared to generate a model classification to distinguish melanoma and lymphocyte cells (Fig. 2b), here plotted using the two most significant differential protein peaks $m / z 6,549$ and 6,079. The correlations between changes in protein features and survival within stage III melanoma patients is evaluated (Fig. 2c), where a decrease in survival correlates with an increase in the relative abundance of the protein at $m / z 6,079$. 


\section{Outlook}

The appeal of MALDI IMS is its capability for acquisition of proteomic data directly from the cellular regions of interest. With current commercial interest improving the matrix deposition, sample acquisition, and data analysis, MALDI IMS is well positioned to gain wider appeal. Recent work in tissue derivatization has opened new doors, where virtually any reagent can be applied to the tissue prior to image acquisition, all while retaining the spatial integrity of the information. Additionally, site-specific protein information from MALDI IMS is a complement to the high-density proteomic information from separationbased analysis (shotgun, 2D gel) and to the imaging strengths of MRI, PET, and antibodybased imaging which together develop a more complete understanding of biological processes. Development of top-down proteomic methods (the ability to directly identify intact proteins within the mass spectrometer) for in situ MALDI IMS will add tremendous value to this technology.

\section{Acknowledgments}

The authors would like to thank Dr. Mark Kelley for supplying the melanoma tumor samples and Dr. Deming Mi for assistance with the statistical analysis. Support provided from DOD grant \# W81XWH-05-1-0179 and 5R01 GM58008-09.

\section{References}

1. Cornett DS, Reyzer ML, Chaurand P, Caprioli RM. MALDI imaging mass spectrometry: molecular snapshots of biochemical systems. Nat Methods 2007;4:828-833. [PubMed: 17901873]

2. Aerni HR, Cornett DS, Caprioli RM. Automated acoustic matrix deposition for MALDI sample preparation. Anal Chem 2006;78:827-834. [PubMed: 16448057]

3. Skold K, Svensson M, Nilsson A, Zhang X, Nydahl K, Caprioli RM, Svenningsson P, Andren PE. Decreased striatal levels of PEP-19 following MPTP lesion in the mouse. J Proteome Res 2006;5:262-269. [PubMed: 16457591]

4. Crecelius AC, Cornett DS, Caprioli RM, Williams B, Dawant BM, Bodenheimer B. Threedimensional visualization of protein expression in mouse brain structures using imaging mass spectrometry. J Am Soc Mass Spectrom 2005;16:1093-1099. [PubMed: 15923124]

5. Andersson M, Groseclose MR, Deutch AY, Caprioli RM. Nat Methods 2008;5:101-108. [PubMed: 18165806]

6. Sinha TK, Khatib-Shahidi S, Yankeelov TE, Mapara K, Ehtesham M, Cornett DS, Dawant BM, Caprioli RM, Gore JC. Integrating spatially resolved three-dimensional MALDI IMS with in vivo magnetic resonance imaging. Nat Methods 2007;5:57-59. [PubMed: 18084298]

7. Khatib-Shahidi S, Andersson M, Herman JL, Gillespie TA, Caprioli RM. Direct molecular analysis of whole-body animal tissue sections by imaging MALDI mass spectrometry. Anal Chem 2006;78:6448-6456. [PubMed: 16970320]

8. Han J, Schey KL. MALDI tissue imaging of ocular lens alpha-crystallin. Invest Ophthalmol Vis Sci 2006;47:2990-2996. [PubMed: 16799044]

9. Oppenheimer, SR. A molecular assessment of tumor margins by MALDI mass spectrometry in renal cell carcinoma. Vanderbilt University; Nashville: 2006.

10. Groseclose MR, Andersson M, Hardesty WM, Caprioli RM. Identification of proteins directly from tissue: in situ tryptic digestions coupled with imaging mass spectrometry. J Mass Spectrom 2007;42:254-262. [PubMed: 17230433]

11. Lemaire R, Desmons A, Tabet JC, Day R, Salzet M, Fournier I. Direct analysis and MALDI imaging of formalin-fixed, paraffin-embedded tissue sections. J Proteome Res 2007;6:1295-1305. [PubMed: 17291023]

12. Schwartz SA, Weil RJ, Thompson RC, Shyr Y, Moore JH, Toms SA, Johnson MD, Caprioli RM. Proteomic-based prognosis of brain tumor patients using direct-tissue matrix-assisted laser desorption ionization mass spectrometry. Cancer Res 2005;65:7674-7681. [PubMed: 16140934] 
13. Cornett DS, Mobley JA, Dias EC, Andersson M, Arteaga CL, Sanders ME, Caprioli RM. A novel histology-directed strategy for MALDI-MS tissue profiling that improves throughput and cellular specificity in human breast cancer. Mol Cell Proteomics 2006;5:1975-1983. [PubMed: 16849436] 
a $\quad \begin{aligned} & \text { Lymph Node w/ Tumor } \\ & \text { Hematoxylin/Eosin (H\&E) }\end{aligned}$

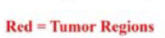

Matrix Spot Array
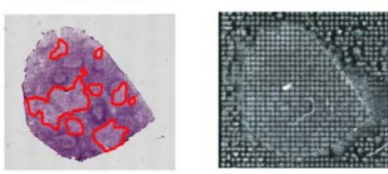

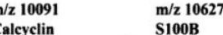

$\mathrm{m} / \mathbf{2} 11643$

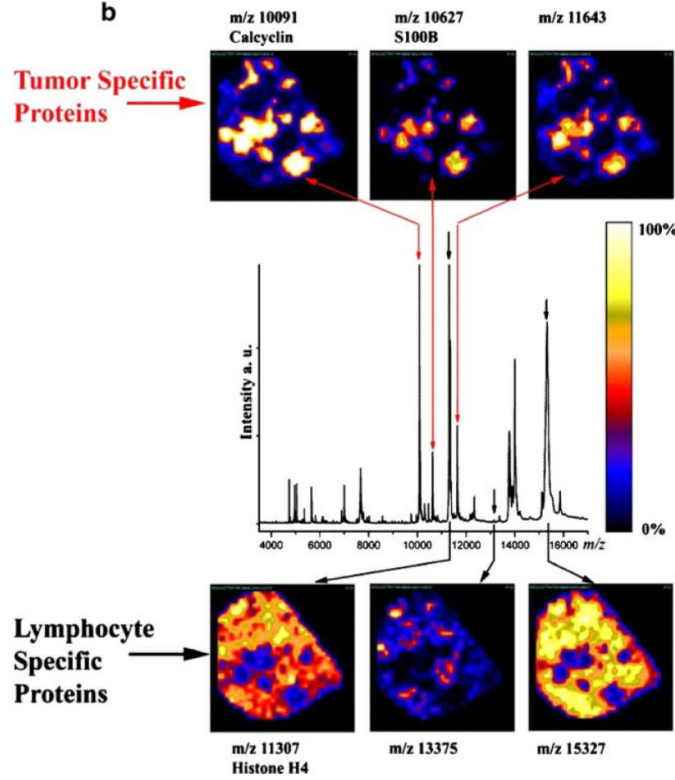

Fig. 1.

MALDI IMS applied to a stage III metastatic melanoma invading the lymph node. a Optical hemotoxylin/eosin stain is shown with the tumor regions outlined in red. Matrix was robotically deposited at $150-\mu \mathrm{m}$ spacing. b Ion density images of proteins with higher observed intensities in the tumor and normal lymphocyte regions are shown. See text for protein identification workflow 

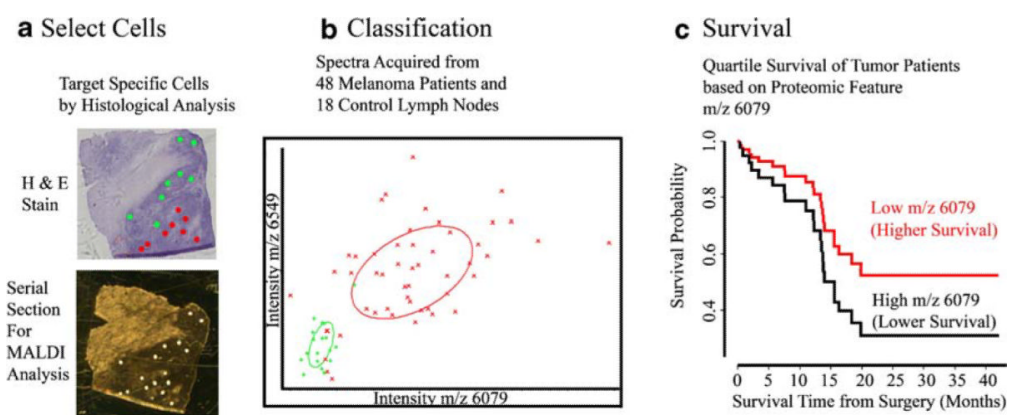

Fig. 2.

Histology directed MALDI profiling of 48 stage III metastatic melanomas and 18 control lymph node tissues. a Cell targeting of $\mathrm{H} \& \mathrm{E}$ stain gives coordinates for MALDI analysis. b Classification of tumor and control cells based on two protein peaks $(\mathrm{m} / \mathrm{z} 6,549$ and 6,079$)$. $\mathbf{c}$ Quartile survival plot in months of melanoma patients with changing intensity of peak $\mathrm{m} / \mathrm{z}$ 6,079 